\title{
TESLA SUPERCONDUCTING ACCELERATING STRUCTURES
}

\author{
J. Sekutowicz \\ Deutsches Elektronen-Synchrotron (DESY), Notkestrasse 85, 22603 Hamburg, Germany
}

Superconducting standing wave structures have been used for charged particle acceleration for almost forty years. A brief introduction to this application with examples, test procedures and recently achieved results are discussed in this article.

\section{INTRODUCTION}

The application of the superconductivity phenomenon in accelerators originated with pioneer work in the late sixties at the High Energy Physics Laboratory (HEPL) at Stanford University [1]. Starting with the deposition of lead on copper and niobium on copper, by the end of 1968 the HEPL group had developed and tested the first bulk niobium superconducting accelerating cavity [2]. The result was very encouraging and initiated R\&D programs at many other laboratories like Brookhaven National Laboratory and Kernforschungszentrum Karlsruhe.

Since then, many superconducting accelerators have been built for the high energy (HEP) and nuclear physics (NP) experiments, both with hadrons and leptons. Table 1 shows the largest superconducting (sc) accelerators for this purpose which have either been operated in the past, at present or are being commissioned for future operation. In the second column (C) stands for circular and (L) for the linear type accelerator, the third column displays the particles being accelerated, while the fourth column lists the final beam energy and the fifth column the number of accelerating cavities ${ }^{1}$ installed in an accelerator.

Table 1: Large sc accelerators for HEP and NP

\begin{tabular}{|c|c|c|c|c|c|}
\hline Name & Type & Particles & $\begin{array}{c}\text { Energy } \\
{[\mathrm{GeV}]}\end{array}$ & $\begin{array}{c}\text { Number of } \\
\text { cavities }\end{array}$ & Status \\
\hline Tristan $^{2}$ & $\mathrm{C}$ & $\mathrm{e}^{+} \mathrm{e}^{-}$ & $2 \times 2$ & 32 & Dismantled \\
\hline LEP $^{3}$ & $\mathrm{C}$ & $\mathrm{e}^{+} \mathrm{e}^{-}$ & $2 \times 100$ & 288 & Dismantled \\
\hline CEBAF $^{4}$ & $\mathrm{~L}$ & $\mathrm{e}^{-}$ & 6 & 320 & Operating \\
\hline HERA $^{5}$ & $\mathrm{C}$ & $\mathrm{e}^{+}$or $\mathrm{e}^{-}$ & 27 & 16 & Operating \\
\hline SNS $^{6}$ & $\mathrm{~L}$ & $\mathrm{H}^{-}$ & 1 & 81 & Commissioning \\
\hline
\end{tabular}

Fifteen years of worldwide development of the technology for the TESLA ${ }^{7}$ linear collider led to many new proposed facilities based on superconducting accelerators (Table 2). Two big projects, the International Linear Collider (ILC) [9] and the European XFEL [10], are continuations of the TESLA collider and its XFEL facility respectively. Both will operate in pulse mode with

\footnotetext{
1 "Cavity" will be used in this paper as the synonym for "structure" and vice versa.

${ }^{2}$ KEK, Tsukuba, Japan [3]

${ }^{3}$ CERN, Geneva, Switzerland [4]

4 TJNAF, Newport News, USA [5]

5 DESY, Hamburg, Germany [6]

${ }^{6}$ ORNL, Knoxville, USA [7]

${ }^{7}$ TeV Energy Superconducting Linear Accelerator [8]
}

a low duty factor of less than one percent. Potential application of superconducting accelerators as drivers for continuous wave operating coherent light sources (Free Electron Lasers) and synchrotron radiation sources results in many other projects worldwide. Three of them are listed in the table. All five future projects have been based on superconducting accelerating cavities designed in 1992 for the TESLA collider [14]. The features of the TESLA cavity will be introduced to the reader in the next section.

Table 2: Proposed future linear sc facilities

\begin{tabular}{|c|c|c|c|l|}
\hline Name & Particles & $\begin{array}{c}\text { Energy } \\
{[\mathrm{GeV}]}\end{array}$ & $\begin{array}{c}\text { Number of } \\
\text { cavities }\end{array}$ & Status \\
\hline ILC & $\mathrm{e}^{+} \mathrm{e}^{-}$ & $2 \times 250$ & 15300 & Studies \\
\hline European XFEL & $\mathrm{e}^{-}$ & 20 & 928 & Proposal \\
\hline ERL [11] & $\mathrm{e}^{-}$ & 5.3 & 310 & Proposal \\
\hline BESSY FEL [12] & $\mathrm{e}^{-}$ & 2.3 & 144 & Proposal \\
\hline 4GLS [13] & $\mathrm{e}^{-}$ & 0.9 & 40 & Proposal \\
\hline
\end{tabular}

\section{TESLA SUPERCONDUCTING CAVITY}

Figure 1 shows the cross-section and a photograph of the 9-cell TESLA structure. The cavity is made of niobium, a type II superconductor, with a critical temperature $T_{\mathrm{c}}=9.2$ $\mathrm{K}$ and a critical magnetic flux $\boldsymbol{B}_{\mathrm{c}} \approx 184 \mathrm{mT}$. The body of the cavity, 9-cells, which from the RF point of view are nine coupled resonators, is cylindrically symmetric. The accelerated beam traverses the cavity along its symmetry axis. The beam tubes at either end have symmetry
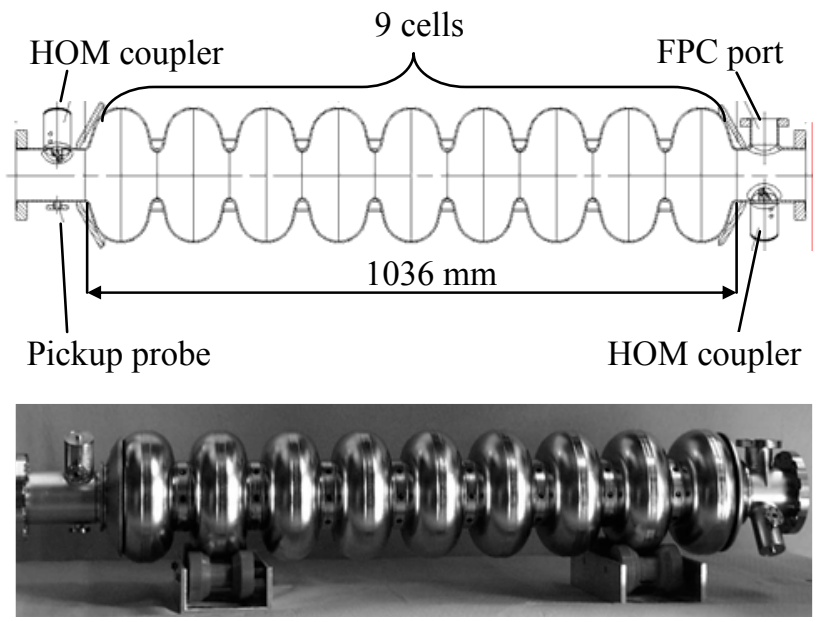

Figure 1: Cross-section and photograph of the superconducting 9-cell TESLA structure equipped with two HOM couplers, a fundamental power coupler (FPC) port and a pickup probe. 
Perturbations introduced by the attached auxiliaries: Higher Order Mode (HOM) couplers, a fundamental power coupler (FPC) and the pickup probe. The axial length of each cell is equal to half of the free space wavelength of the fundamental (accelerating) resonant mode. At the operating TESLA frequency of $1.3 \mathrm{GHz}$ the cell length is $\lambda / 2=115.3 \mathrm{~mm}$.

\section{Interaction with accelerated beam ${ }^{8}$}

Figure 2 shows computed magnetic and electric field contours for the accelerating mode. The structure is a so called standing wave structure in which particles experience the Lorentz force of the standing electromagnetic wave. As known, $n$ coupled resonators can oscillate in $n$ modes for each resonance field pattern being an eigenvector of a single (uncoupled) cell. The $n$ modes, which differ in frequency and cell-to-cell phase advance, form the pass-band. In the TESLA structure the $\mathrm{TM}_{010}$ like ${ }^{9}$ resonance pattern is utilized for particle acceleration. It is a monopole mode with no angular dependency of the fields inside the cylindrical symmetric part of the cavity and it is the highest frequency mode in the $\mathrm{TM}_{010}$ passband. The cell-to-cell phase advance is $\pi$. When the structure is well tuned all cells have the same amplitude $|\boldsymbol{E}|$ of the accelerating electric field on the axis (Fig. 3).

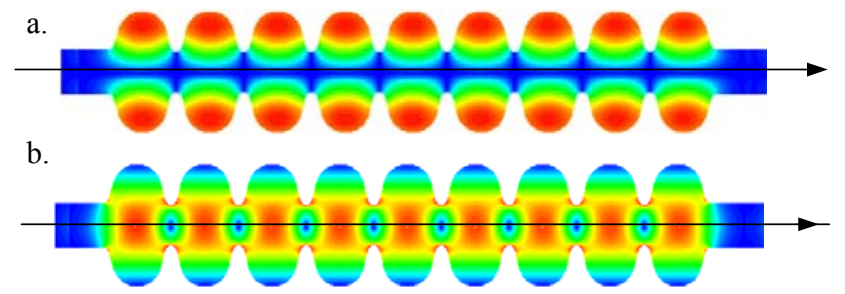

Figure 2: Contour of magnetic (a) and electric (b) field in the TESLA structure. On the color scale red means high and blue low value respectively.

In general, the interaction between the fundamental mode of a cavity and a charged beam may lead to two processes:

- Beam energy gain - acceleration

- Beam energy loss - deceleration, energy recovery process.

Furthermore, the beam may excite other resonances, HOMs of a cavity:

- HOM excitation.

The excited HOM's very often degrade beam quality causing emittance growth and/or energy modulation.

\footnotetext{
${ }^{8}$ The whole material presented in the following subsections applies in general to any superconducting standing wave accelerating structure.

9 The convention to name field patterns follows the convention commonly used for cylindrical waveguides and cylindrical RF resonators [15] for cylindrical coordinate system $(\varphi, r, z)$. TM stands for transverse magnetic (no z-component of magnetic field). Indices 010 refer to the number of half wave length in all three dimensions. 0 means field has constant value in the direction. 1 means a half wavelength in the direction and so on. The convention, when applied to cylindrical resonators with no openings (irises), is very helpful to describe the field pattern of a mode, but it is slightly misleading in case of cells of an accelerating structure, which fields vary in z-direction.
}

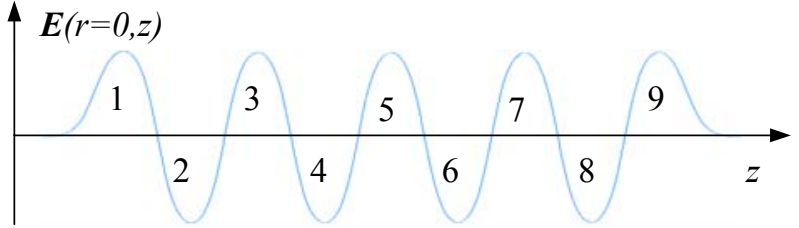

Figure 3: Computed normalized accelerating electric field on the axis in the TESLA structure.

Interaction between the electric field of a mode $k$ and a point-like charge, having relativistic velocity close to the speed of light $c$, is described in the frequency domain by the characteristic impedance $(R / Q)_{k}^{10}$ :

$$
(R / Q)_{k} \equiv \frac{\left|V_{k}\right|^{2}}{\omega_{k} W_{k}}
$$

where $\omega_{k}=2 \pi f_{k}$ and $W_{k}$ are the angular frequency of the mode and its stored energy respectively. $\left|\boldsymbol{V}_{k}\right|$ is the amplitude of the voltage experienced by the bunch of particles along its trajectory:

$\left|\boldsymbol{V}_{k}\right|=\sqrt{\left(\int_{y_{o}}^{l} \overline{\boldsymbol{E}}_{k} \sin \left(\frac{\omega_{k}}{c}\left(y-y_{0}\right)\right) d y\right)^{2}+\left(\int_{y_{o}}^{l} \overline{\boldsymbol{E}_{k}} \cos \left(\frac{\omega_{k}}{c}\left(y-y_{0}\right)\right) d y\right)^{2}}$

$l$ and $y_{0}$ are the trajectory length and its beginning respectively, $\overline{\boldsymbol{E}}_{k}$ is the electric field along the trajectory.

The higher $(R / Q)_{k}$ the stronger is the potential energy exchange between the mode and the bunch. The way the voltage $\left|\boldsymbol{V}_{k}\right|^{11}$ is defined makes $(R / Q)_{k}$ independent of the phase a bunch enters the cavity. The net energy exchanged $\Delta U_{k}$ with the mode is:

$$
\Delta U_{k} \equiv|q| V_{k}\left|\cos \left(\varphi_{k}-\varphi_{0, k}\right)\right|
$$

which depends on the entrance phase $\varphi_{k}$, the charge of the bunch $q$ and the mode stored energy $W_{k}$ since $\left|\boldsymbol{V}_{k}\right| \approx \sqrt{ } W_{k}$. The phase $\varphi_{0, k}$ giving the maximum $\Delta U_{k}$ is defined by the equation:

$$
\varphi_{0, k}=\arcsin \left(\frac{\int_{y_{o}}^{l} \overline{\boldsymbol{E}}_{k} \cos \left(\frac{\omega_{k}}{c}\left(y-y_{0}\right)\right) d y}{\left|\boldsymbol{V}_{k}\right|}\right)
$$

Figure 4 shows the simplified replacement lumped element circuit for the interaction of a beam with the fundamental mode (beam loading). The LCR circuit represents the accelerating structure with its intrinsic

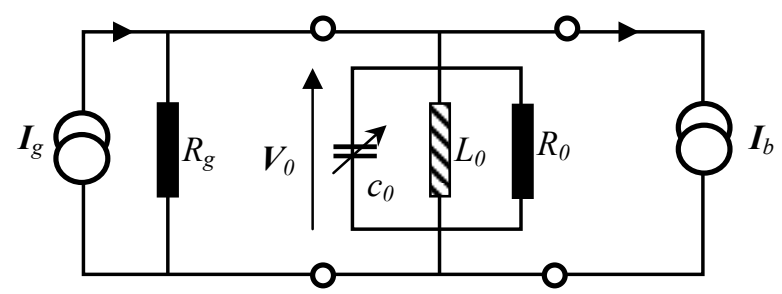

Figure 4: Replacement circuit for the beam loading

\footnotetext{
${ }^{10}$ In this article (R/Q) is defined in the linac convention. For the RF definition one needs to take half of its value.

${ }^{11}$ Italic bold characters denote phasors
} 
resistance $R_{o}$. The beam is represented by the current source $\boldsymbol{I}_{b}$. The RF power source has been replaced by a current source $\boldsymbol{I}_{g}$ and its impedance $R_{g}$. The impedances are defined as follows:

$$
\begin{gathered}
R_{0}=(R / Q)_{F M} Q_{0} \\
R_{g}=(R / Q)_{F M} Q_{e x t}
\end{gathered}
$$

$(R / Q)_{F M}$ is the characteristic impedance of the fundamental mode. $Q_{o}$ and $Q_{e x t}$ are the intrinsic and external quality factors. The power $P_{b}$ delivered to or extracted from the beam and power dissipated in the cavity wall $P_{0}$ are:

$$
\begin{gathered}
P_{b} \equiv\left|\boldsymbol{V}_{0}\right|\left|\boldsymbol{I}_{b}\right| \cos \left(\Phi_{b}\right) \\
P_{0} \equiv \frac{\left|\boldsymbol{V}_{\boldsymbol{0}}\right|^{2}}{R_{0}}
\end{gathered}
$$

where $\Phi_{b}$ is the phase between the beam current $\boldsymbol{I}_{b}$ and the cavity voltage $\boldsymbol{V}_{0}$. The phases of the voltages and currents from the replacement circuit are shown in Figure 5. At the resonance frequency and without the beam the generator voltage $\boldsymbol{V}_{g}$ and its current $\boldsymbol{I}_{g}$ are parallel, which means that the RF source is loaded by real impedance and that energy transfer from the RF source to the load is most effective. In general when particles traverse the cavity the beam induced voltage $\boldsymbol{V}_{b}$ "detunes" the cavity [16]. The vector sum:

$$
\boldsymbol{V}_{\boldsymbol{0}}^{\prime}=\boldsymbol{V}_{g}+\boldsymbol{V}_{b}
$$

is not parallel to $\boldsymbol{I}_{g}$ and a correction of the cavity frequency must take place to rotate this vector by the angle $-\Delta \Phi$ :

$$
\Delta \Phi \equiv \arctan \frac{\left|\boldsymbol{V}_{\boldsymbol{b}}\right| \sin \left(\Phi_{b}\right)}{\left|\boldsymbol{V}_{\boldsymbol{g}}\right|-\left|\boldsymbol{V}_{b}\right| \cos \left(\Phi_{b}\right)}
$$

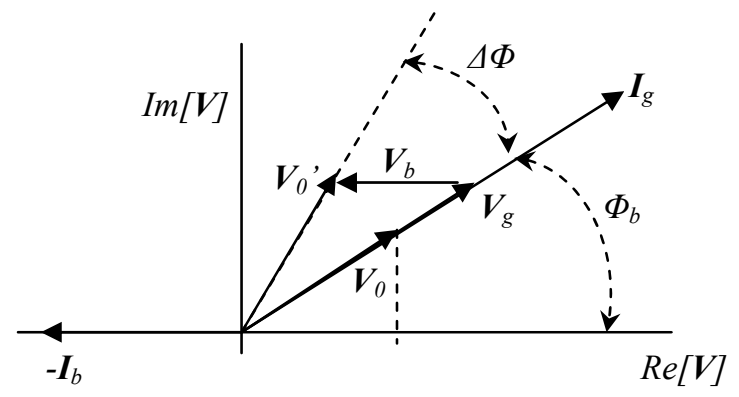

Figure 5: Phases for the replacement circuit

Practically, in circular accelerators like synchrotrons, $\Phi_{b}$ varies between $90^{\circ}$ at the injection and $45^{\circ}$ when beam is stored for experiments. In linear accelerators $\Phi_{b}$ is close to 0 . The acceleration is "on crest" and $\Delta \Phi$ is 0 or

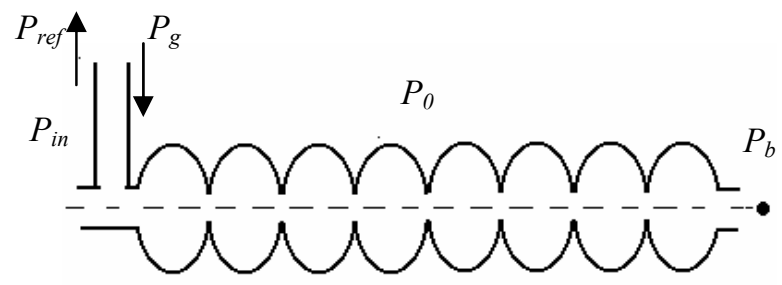

Figure 6: Power distribution scheme in a standing wave structure. very small. Schematic RF power distribution for a standing wave structure accelerating the beam is shown in Fig. 6. The input power $P_{\text {in }}$ is:

$$
P_{i n}=P_{0}+P_{b}=P_{f o r}-P_{r e f}=\frac{4 \beta_{b}}{\left(1+\beta_{b}\right)^{2}} P_{f o r}
$$

$P_{\text {for }}$ and $P_{\text {ref }}$ are the incident and the reflected power respectively. $\beta_{b}$ is the dynamic, beam dependent coupling factor between the cavity and input line:

$$
\beta_{b}=\frac{\beta_{c}}{\left[1+\frac{Q_{0}\left|\boldsymbol{I}_{b}\right|(R / Q)_{F M} \cos \left(\Phi_{b}\right)}{\left|\boldsymbol{V}_{\boldsymbol{0}}\right|}\right]}
$$

$\beta_{c}$ is the intrinsic wall loss dependent coupling factor:

$$
\beta_{c}=\frac{Q_{0}}{Q_{e x t}} .
$$

The amplitudes of $\boldsymbol{V}_{g}$ and $\boldsymbol{V}_{b}$ are given by:

$$
\begin{gathered}
\left|\boldsymbol{V}_{g}\right|=\sqrt{\frac{4 \beta_{c}}{\left(1+\beta_{c}\right)^{2}} P_{f o r}(R / Q) Q_{o}} \\
\left|\boldsymbol{V}_{b}\right|=\left|\boldsymbol{I}_{b}\right|(R / Q) \frac{Q_{o}}{\left(1+\beta_{c}\right)}
\end{gathered}
$$

The optimum external quality factor at which the operation is reflection-free $\left(P_{r e f}=0\right)$ is achieved when $Q_{e x t}$ fulfils the following condition:

$$
Q_{\text {ext }}=\frac{Q_{0}}{1+\frac{Q_{0}\left|\boldsymbol{I}_{b}\right|(R / Q)_{F M} \cos \left(\Phi_{b}\right)}{\left|\boldsymbol{V}_{\boldsymbol{0}}\right|}} .
$$

\section{Intrinsic wall losses}

A time-dependent RF magnetic field applied to a superconductor dissipates energy in a thin layer (the penetration depth). This is different from the DC case, where currents flow without losses. The dissipation is due to the temperature dependent $\mathrm{BCS}^{12}$ resistance $r_{B C S}$ and due to impurities in the superconductor resulting in the residual resistance $r_{r}$. The total surface resistance $r_{s}$ for niobium in the superconducting state at the temperature $T$ and the frequency $f$ is:

$$
r_{s}=r_{r}+r_{B C S}=r_{r}+\frac{0.0002}{T}\left(\frac{f[G H z]}{1.5}\right)^{2} \exp \left(-\frac{17.67}{T}\right)
$$

In the case of the TESLA cavity $r_{B C S}$ is $\sim 10 \mathrm{n} \Omega$ at $T=2 \mathrm{~K}$. For a very pure niobium e.g. big grain or single crystal material, for which $r_{r}$ is of the order of $1 \mathrm{n} \Omega$ an operation at $1.8 \mathrm{~K}$ or even $1.7 \mathrm{~K}$ can be economically justified, even though it requires more complex refrigeration.

The dissipated power $P_{k}$ by mode $k$ in the cavity wall is:

$$
P_{k} \equiv \frac{r_{s}}{2} \int_{S}\left|\overline{\boldsymbol{H}}_{k}\right|^{2} d s
$$

where $\overline{\boldsymbol{H}}_{k}$ is the magnetic field of mode $k$ on the wall and $S$ denotes the inner surface of a cavity.

\footnotetext{
12 BCS stands for J. Bardeen, L. Cooper and R. Schrieffer. Their theory explains phenomenon of superconductivity (Nobel price in 1972).
} 
The geometric factor $G_{k}$ :

$$
G_{k} \equiv \frac{\omega_{k} \cdot W_{k}}{\frac{1}{2} \int_{S}\left|\overline{\boldsymbol{H}}_{k}\right|^{2} d s}
$$

is commonly used to compare various cell shapes with respect to their cryogenic losses. The higher $G_{k}$ the lower the dissipation $P_{k}$ at the given stored energy $W_{k}$ and surface resistance $r_{s}$. The above definition of $G_{k}$ yields to the relation:

$$
G_{k}=Q_{k} \cdot r_{s}=\frac{\omega_{k} \cdot W_{k} \cdot r_{s}}{P_{k}}
$$

Consequently, higher $G_{k}$ of a mode $k$ means higher intrinsic quality factor $Q_{k}$.

The dissipated power $P_{0}$ for the fundamental mode at the accelerating voltage $\left|\boldsymbol{V}_{0}\right|$ can now be expressed by the formula:

$$
P_{0}=\frac{r_{s} \cdot\left|V_{0}\right|^{2}}{G_{F M} \cdot(R / Q)_{F M}}
$$

which indicates that cell shapes with high product $G_{F M}(R / Q)_{F M}$ are preferable due to the lower dissipation.

\section{Maximum Fields on the Cavity Wall}

The magnetic flux on the cavity wall has to be smaller than $B_{\mathrm{c}}$, a critical value at which superconductivity breaks and niobium goes to the normal-conducting state. The ratio:

$$
\eta_{B} \equiv \frac{\left|\boldsymbol{B}_{\text {peak }}\right|}{\left|\boldsymbol{E}_{\text {acc }}\right|}
$$

of the peak magnetic flux $\left|\boldsymbol{B}_{\text {peak }}\right|$ on the wall to the accelerating gradient $\left|\boldsymbol{E}_{a c c}\right|$ :

$$
\left|E_{\text {acc }}\right| \equiv \frac{\left|V_{0}\right|}{l_{\text {active }}}
$$

where $l_{\text {active }}$ is the axial length of all cells, shows the ultimate limit in the achievable gradient for the shape.

The ratio of the peak electric field on the wall $\left|\boldsymbol{E}_{\text {peak }}\right|$ to $\left|\boldsymbol{E}_{\text {acc }}\right|$ :

$$
\eta_{E} \equiv \frac{\left|\boldsymbol{E}_{\text {peak }}\right|}{\left|\boldsymbol{E}_{\text {acc }}\right|}
$$

has practical meaning. The fields on the wall can be as high as $100 \mathrm{MV} / \mathrm{m}$. Such a strong field withdraws electrons from sharp edges of grain boundaries, welds or residual particulates on the surface. When these electrons accelerated by the electric field they either bombard the superconducting wall of the cavity or get captured into synchronic acceleration forming dark currents bombarding surface of other cavity in a cavity chain. Both processes lead to $\mathrm{x}$-rays which may cause a quench, a local loss of superconductivity transferring the whole stored energy into heat load. The design of the TESLA cavity in 1992 assumed this phenomenon as the potential limit in the performance and much effort was done to keep this factor close to two.

Very recently, due to significant progress in the surface preparation methods over the past fifteen years, it was proposed to optimize the ILC cavity shape with respect to $\eta_{B}$. Two proposed shapes will be briefly discussed later but both have $\eta_{E}>2$ and seem to be more sensitive to residual impurities on the surface.

\section{Cell-to-cell Coupling}

The cell-to-cell coupling $k_{c c}$ for the fundamental mode in a $N$-cell structure plays an important role for the field profile sensitivity to cell frequency errors. The sensitivity factor $a_{f f}$ is defined in the following way [17]:

$$
a_{f f}=\frac{N^{2}}{k_{c c}}
$$

The relative sensitivity of the field amplitude $A_{\mathrm{i}}$ in cells is given by the equation:

$$
\frac{\Delta A_{i}}{A_{i}}=a_{f f} \frac{\Delta f_{i}}{f}
$$

where $\Delta f_{i}$ is the frequency error of the cell number $i . k_{c c}$ increases with radius of the iris but this makes $\eta_{E}$ and $(R / Q)_{F M}$ less favorable.

\section{Cell Shape Optimization}

In general, the shape optimization is a difficult process because an improvement in one parameter causes worsening of others [18]. One varies the geometric parameters (GP) shown in Fig. 7 to shape the inner cells. The most common criteria for optimization and the RFparameters, which have to be optimized for a criterion, are listed in the first and second column of Table 3. In the table ( $\mathbf{D} \downarrow)$ and (I $\uparrow$ ) indicate decreasing and increasing value respectively. The third column shows required change in an RF-parameter. The iris radius $r_{\mathrm{i}}$ is the most effective geometric parameter for each criterion (see last column). Other GPs listed in the last column are used for fine tuning.

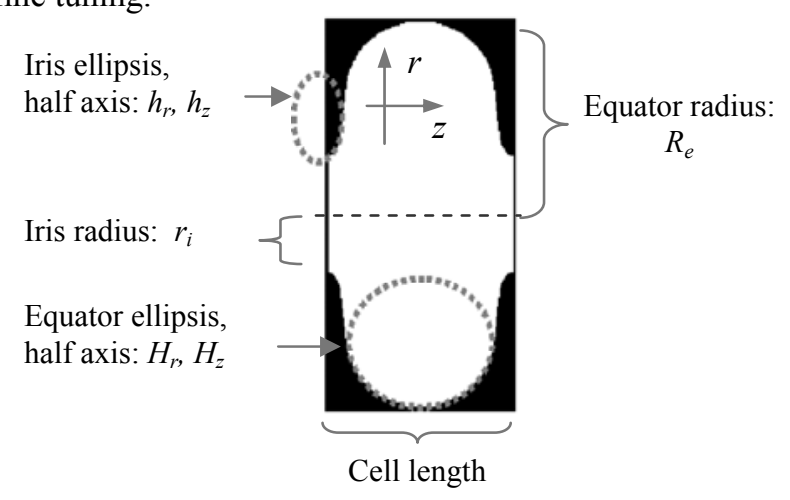

Figure 7: Geometrical parameters of a cell.

Table 3: Criteria and RF-parameters

\begin{tabular}{|c|c|c|c|}
\hline Criterion & RF-parameter. & Opt. & GP \\
\hline High gradient & $\eta_{B}$ and $\eta_{E}$ & $\mathbf{D} \downarrow$ & $\begin{array}{c}r_{i} \leftrightarrow(\mathbf{D} \downarrow) \\
h_{r}, h_{z}, H_{r}, H_{z}\end{array}$ \\
\hline Low cryogenic load & $(R / Q)_{F M} G_{F M}$ & $\mathbf{I} \uparrow$ & $\begin{array}{c}r_{i} \leftrightarrow(\mathbf{D} \downarrow) \\
H_{r}, H_{z}\end{array}$ \\
\hline Low HOM impedance & $(R / Q)_{H O M}$ & $\mathbf{D} \downarrow$ & $r_{i} \leftrightarrow(\mathbf{I} \uparrow)$ \\
\hline
\end{tabular}




\section{Parameters of the TESLA and ILC Structures}

The RF-properties of a multi-cell cavity are usually dominated by those of the inner cells. This is the case found in the TESLA cavities. Tables 4 and 5 show RFparameters of the inner cell and the whole TESLA structure respectively. In addition, in Table 5 the nominal operation parameters for the ILC are displayed in the last four rows. These parameters are still under discussion and in the end may differ from those displayed here. The inner cell shape is shown in Figure 8 along with two alternative shapes proposed recently (Table 6). The first, Low Loss (LL) shape was first proposed in 2002 for the $12-\mathrm{GeV}$ upgrade of the CEBAF accelerator [19] and was later scaled for the ILC frequency [20]. The iris of the LL cell is $60 \mathrm{~mm}$ in diameter as compare to $70 \mathrm{~mm}$ of the original TESLA cell. This allows for lower $\eta_{B}$ and enhances the limit in the achievable gradient to $51 \mathrm{MV} / \mathrm{m}$ from 44 $\mathrm{MV} / \mathrm{m}$ for the TESLA shape. High $(R / Q)_{F M}$ and $G_{F M}$

Table 4: Parameters of the inner cell

\begin{tabular}{|l|l|l|}
\hline$f_{F M}$ & {$[\mathrm{MHz}]$} & 1300 \\
\hline$k_{c c}$ & {$[\%]$} & 1.98 \\
\hline$\eta_{E}$ & - & 1.98 \\
\hline$\eta_{B}$ & {$[\mathrm{mT} /(\mathrm{MV} / \mathrm{m})]$} & 4.15 \\
\hline$(R / Q)_{F M}$ & {$[\Omega]$} & 113.8 \\
\hline$G_{F M}$ & {$[\Omega]$} & 271 \\
\hline$(R / Q)_{F M} G_{F M}$ & {$\left[\Omega^{2}\right]$} & 30840 \\
\hline
\end{tabular}

Table 5: Parameters of the TESLA cavity

\begin{tabular}{|l|l|l|}
\hline$f_{F M}$ & {$[\mathrm{MHz}]$} & 1300 \\
\hline$(R / Q)_{F M}$ & {$[\Omega]$} & 1012 \\
\hline$G_{F M}$ & {$[\Omega]$} & 271 \\
\hline$l_{\text {active }}$ & {$[\mathrm{mm}]$} & 1038 \\
\hline$a_{f f}$ & - & 4091 \\
\hline$Q_{\text {ext }}$ & {$\left[10^{6}\right]$} & 3.4 \\
\hline$Q_{0}$ at $\boldsymbol{E}_{a c c}=31.5 \mathrm{MV} / \mathrm{m}$ & {$\left[10^{10}\right]$} & 1 \\
\hline Nominal gradient $\left|E_{a c c}\right|$ & {$[\mathrm{MV} / \mathrm{m}]$} & 31.5 \\
\hline$P_{\text {in }}$ & {$[\mathrm{kW}]$} & 312 \\
\hline$I_{b}$ & {$[\mathrm{~mA}]$} & 9.5 \\
\hline$\Phi_{b}$ & {$\left[{ }^{\circ}\right]$} & 0 \\
\hline
\end{tabular}

a)

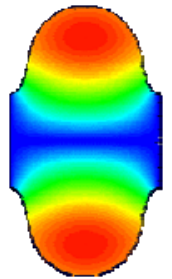

b)

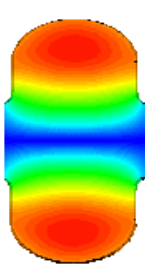

c)

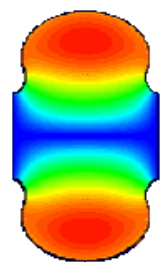

Figure 8: Magnetic field contour of the fundamental mode in inner cells proposed for the ILC, a) TESLA, b) Low Loss, c) Re-entrant. lead to significantly higher combined product which results in a $\sim 20 \%$ reduction in the cryogenic load in comparison to the TESLA cell. On the other hand smaller $k_{c c}$ and higher $\eta_{E}$ made that shape more sensitive to cell frequency errors and increased probability of the electron emission phenomenon. Similar, the Re-entrant cavity allows for higher gradients [21, 22]. It has more favorable $\eta_{E}$ and $k_{c c}$ as compared to the LL shape, but technical difficulty in acid and water removal after the cleaning in a multi-cell assembly might limit its applications.

Table 6: Alternative inner cell shapes

\begin{tabular}{|l|l|l|l|}
\hline & \multicolumn{1}{|c|}{ Unit } & LL & RE \\
\hline$f_{F M}$ & {$[\mathrm{MHz}]$} & 1300 & 1300 \\
\hline$k_{c c}$ & {$[\%]$} & 1.52 & 1.8 \\
\hline$\eta_{E}$ & - & 2.36 & 2.21 \\
\hline$\eta_{B}$ & {$[\mathrm{mT} /(\mathrm{MV} / \mathrm{m})]$} & 3.61 & 3.76 \\
\hline$(R / Q)_{F M}$ & {$[\Omega]$} & 133.7 & 126.8 \\
\hline$G_{F M}$ & {$[\Omega]$} & 284 & 277 \\
\hline$(R / Q)_{F M} G_{F M}$ & {$\left[\Omega^{2}\right]$} & 37970 & 35120 \\
\hline
\end{tabular}

\section{VERTICAL TEST}

The economically justified and therefore commonly used fabrication technique of elliptical $\mathrm{Nb}$ cavities by deep-drawing of metal sheets and electron beam welding of half-cells has a severe draw-back. The shapes of built cavities deviate from their RF theoretical models and the frequencies of individual cells in a multi-cell cavity have to be corrected to equalize the field profiles of modes. Unfortunately this is possible only for the accelerating mode and is done by means of plastic deformation of cells. In general, frequencies of HOMs stay perturbed but luckily many of high impedance parasitic modes have strong cell-to-cell coupling and their field profiles still allow for their sufficient damping. Very few high (R/Q) passbands have small cell-to-cell coupling and sensitive field profiles. In this case the modes suppression needs more attention and can be corrected for example by a dedicated adjustment of HOM couplers.

The tuning of the FM field profile has to be done very carefully. The number of plastic deformations must be kept as low as possible because they change mechanical properties of niobium. The difficulty is that in a multi-cell cavity cells stay electromagnetically coupled and modifying the frequency of one cell changes the field profile in all cells. A method based on frequency and field profile measurement for all modes of the FM passband was successfully implemented the first time in the late 80's [23]. All sixteen HERA cavities and more than seventy TESLA cavities have been tuned up to now using that method and no mechanical changes have been observed.

In preparation for the test in a vertical cryostat, after tuning and chemical cleaning, the cavity is equipped with a coaxial input antenna providing very high $Q_{\text {ext }}$, close to an expected $Q_{0}$ of the cavity at the chosen test 
temperature. Usually the input antenna, attached to one of the beam tubes, allows for limited coupling adjustment during the test (variable antenna). In the case of a structure built for the beam operation the port for FPC and HOM coupler output ports are typically blanked off. Both, test cavities and those for beam operation are equipped with field (pickup) probe. In the vertical cryostat the whole cavity under the test is immersed in liquid helium. One should note that in the vertical tests the $3 \mathrm{~dB}$ resonance width is often a fraction of a $\mathrm{Hz}$. To ensure field stability for the measurement, the RF-source has to be locked to the frequency of the tested cavity which is modulated by mechanical resonances and helium pressure variation. This is different from the beam operation, when all cavities in an accelerator must have the same frequency and proper phase, and therefore all are locked to the master oscillator.

The main aim of the vertical tests is to determine the $Q_{0}$ value as a function of $\left|\boldsymbol{E}_{a c c}\right|$. When the input antenna and pickup probe cables are calibrated at the cryogenic temperature the next step is to measure their $Q_{\text {ext }}$. For this, one investigates transmitted and reflected signals which are a response of the tested cavity to a rectangular RF pulse. The amplitude $A(t)$ of the reflected power is shown in Fig. 9. Both solid curves show real reflected signals for an over-critical coupling (red curve) and an under-critical coupling (green curve). Both blue lines show signals displayed by a spectrum analyzer or a scope when the negative sign is dropped. The dot-line shows the displayed signal for the under-critical coupling and the dash-line for the over-critical coupling. When no nonlinear phenomenon takes place (like multipacting, quench, field emission...) the measured signals agree very well with the theoretical model. The functions $f 1(t)$ and $f 2(t)$ in Fig. 9 can be found by means of the Laplace transformation for the previously discussed replacement circuit without the beam. They are defined by the following equations:

$$
\begin{array}{ll}
f 1(t)=-\frac{1-\beta_{L}}{1+\beta_{L}}-\frac{2 \beta_{L}}{1+\beta_{L}} e^{-\frac{\omega_{0} t}{2 Q_{L}}} S(t) & \text { for } t \in<0, \tau_{p}> \\
f 2(t)=f 1(t)+f 1\left(t-\tau_{p}\right) S\left(t-\tau_{p}\right) & \text { for } t \in<\tau_{p}, \infty>
\end{array}
$$

where $S(t)$ is the unit step function, $\tau_{p}$ is the pulse duration, $Q_{L}$ and $\beta_{L}$ are loaded quality factor and coupling factor of the input antenna respectively. From the theoretical model one can compute $\beta_{L}$ knowing reflected signal values at time: $t=0, t=\tau_{p^{-}}$and $t=\tau_{p}+$. Three formulae can be used to define $\beta_{L}$ :

$$
\begin{gathered}
\beta_{L}=\frac{A(0)-A\left(\tau_{p}^{-}\right)}{A(0)+A\left(\tau_{p}\right)} \\
\beta_{L}=\frac{A\left(\tau_{p}+\right)}{2 A(0)-A\left(\tau_{p}+\right)} \\
\beta_{L}=\frac{A\left(\tau_{p^{+}}\right)}{\left.2 A\left(\tau_{p^{-}}\right)\right)+A\left(\tau_{p^{+}}\right)}
\end{gathered}
$$

Practically all three values are computed to find the mean

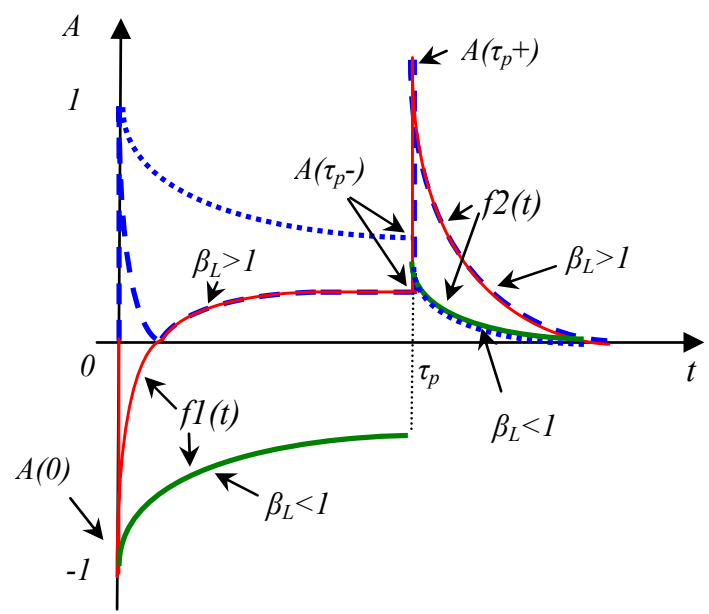

Figure 9: Time depended response to a rectangular RF pulse. Theoretical function $f 1(t)$ describes the amplitude of the reflected power for the pulse duration, $f 2(t)$ after it has been switched off.

value of $\beta_{L}$ with the smallest error. $Q_{L}$ is measured by means of the decay of the field probe signal (transmitted signal, $P_{\text {tran }}$ ) right after the RF pulse is switched off. The cavity stored energy decays exponentially due to the power dissipation and radiation via all active ports:

$$
W(t)=W\left(\tau_{p}+\right) e^{-\frac{\omega_{0}\left(t-\tau_{p}+\right)}{Q_{L}}}
$$

The transmitted power, which is proportional to $W(t)$, when measured at a chosen time interval allows to determine $Q_{L}$. In addition to $Q_{L}, \beta_{L}$ and $P_{\text {tran }}$, the input power $P_{\text {in }}$ needs to be measured to finally determine the intrinsic quality factor $Q_{0}$ :

$$
Q_{0}=Q_{L}\left(1+\beta_{L}\right)\left(1+\frac{P_{\text {tran }}}{P_{\text {in }}-P_{\text {tran }}}\right)
$$

\section{Test Examples; Single-cell Cavities}

The single-cell $1.3 \mathrm{GHz}$ cavities of the LL and RE shape fabricated and tested at KEK in Japan reached the highest accelerating gradient in niobium cavities. The measured curves $Q_{0}$ as a function of $\left|\boldsymbol{E}_{a c c}\right|$ at $1.68 \mathrm{~K}$ and

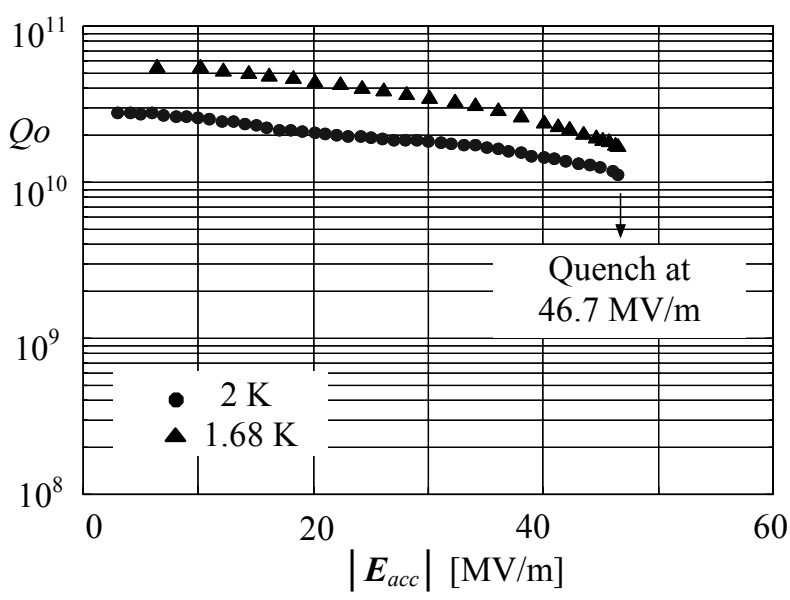

Figure 10: LL single cell cavity as tested at KEK. Test was performed at $2 \mathrm{~K}$ and $1.68 \mathrm{~K}$. Courtesy K. Saito. 
$2 \mathrm{~K}$ for the LL-shape are shown in Fig. 10, where the cavity reached $46.7 \mathrm{MV} / \mathrm{m}$. The highest accelerating gradient of $50.9 \mathrm{MV} / \mathrm{m}$ was achieved for the single-cell $\mathrm{RE}$ shape cavity. The test was done at $2 \mathrm{~K}$ and the intrinsic $Q_{0}$ at that gradient was $7 \cdot 10^{9}$. One should notice that in that test peak magnetic flux on the wall was very close to its critical value for niobium and the peak electric field reached $112 \mathrm{MV} / \mathrm{m}$.

The original TESLA single-cell cavities demonstrated gradients on the order of $40 \mathrm{MV} / \mathrm{m}$, with peak electric fields up to $82 \mathrm{MV} / \mathrm{m}$ and peak magnetic fluxes up to 185 $\mathrm{mT}$. Figure 11 displays the test result obtained recently at DESY for the single-cell TESLA type cavity made of the poly-crystal niobium. This test was performed at $2 \mathrm{~K}$.

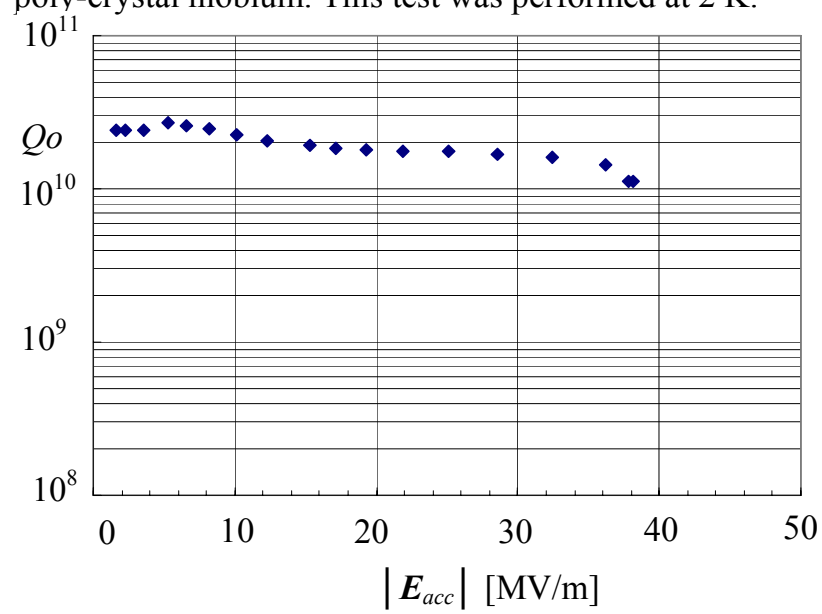

Figure 11: Test result of the single-cell TESLA shape cavity made of the large grain niobium.

All single-cell cavities discussed in this subsection were electropolished (EP). The procedure was first applied in early 70's by the Siemens Company in collaboration with the Kernforschungszentrum Karlsruhe group (P. Kneisel and co-workers). In the mid 80`s the method was applied to the TRISTAN cavities (K. Saito) and since 1998 to the single-cell and multi-cell cavities at all laboratories working on high gradients in elliptical structures (TJNAF, KEK, DESY, CEA-Saclay). The advantage of the EP treatment in the case of the polycrystal niobium is a smoothing of the surface roughness to around $1 \mu \mathrm{m}$. This allows for better removing of emitting residual particulates by the high pressure water rinsing (HPR) procedure which follows the chemical treatment.

\section{Test Examples; 9- cell Cavities}

The excellent single-cell results are not simply reproduced for the multi-cell structures even though they have very similar shape. The difficulties come from the application of the preparation techniques: chemical treatment and HPR to much larger and convoluted surface. In addition a large surface of a multi-cell structure has a much higher probability of defects and contaminations leading to quenching at high gradients.

The Buffered Chemical Polishing (BCP) was the chemical treatment applied to the first, second and third production of the TESLA cavities, many of which still

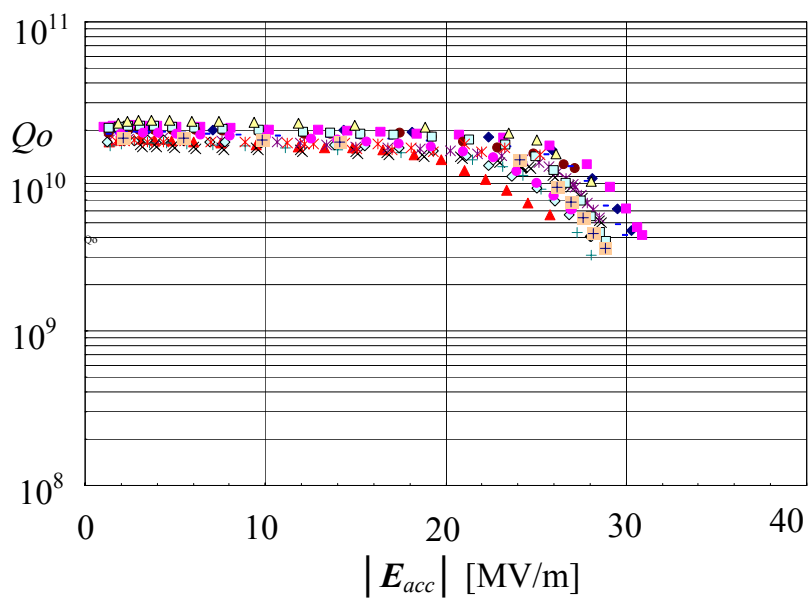

Figure 12: Test result of fourteen 9-cell TESLA cavities cleaned with BCP.

operate in the Tesla Test Facility linac at DESY. The test results for the best $\mathrm{BCP}$ treated cavities from the third production are shown in Fig. 12. Their performance met the very first (1997) specification of the TESLA-500 GeV collider, which was $25 \mathrm{MV} / \mathrm{m}$ and $5 \cdot 10^{9}$ for the accelerating gradient and intrinsic quality factor respectively.

The test results for the best performing four poly-crystal TESLA cavities at DESY, which have been cleaned with the EP method are displayed in Fig. 13. As expected these cavities reached higher gradients and exhibited less radiation as compared to the BCP treated cavities, for which $Q_{0}$ drops due to the heavy electron emission above $25 \mathrm{MV} / \mathrm{m}$. Still one should notice that the BCP process is much simpler and less expensive than the EP treatment. This has significant impact on the capital cost of big accelerators like ILC and the European XFEL linac.

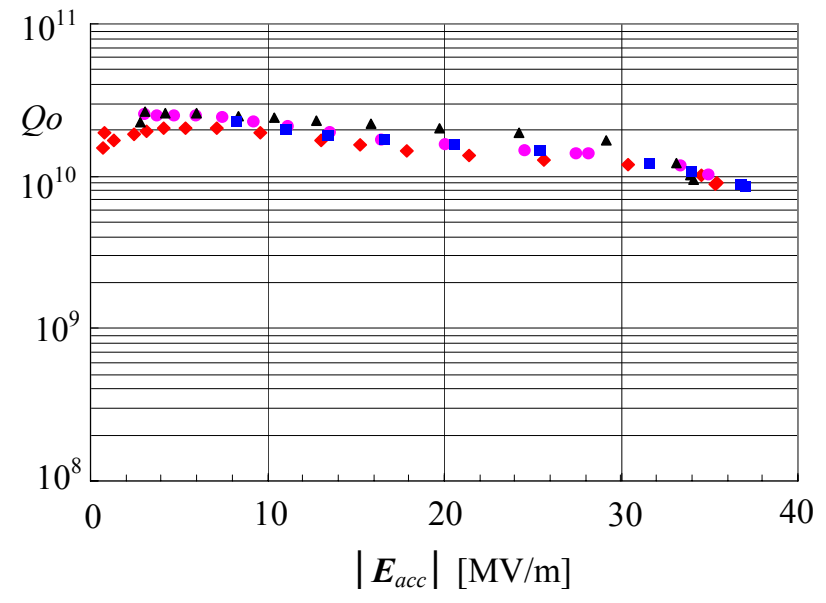

Figure 13: Test result of four 9-cell poly-crystal TESLA cavities cleaned with EP.

\section{Test Examples; Large Grain Single-cell Cavities}

The breakthrough in the cost saving is expected to come from the very recently proposed (TJNAF group) implementation of the large grain niobium (LGNb). Slices for deep drawing were cut directly from the ingot to build several single-cell cavities at higher frequency. The surface of LGNb (and single crystal $\mathrm{Nb}, \mathrm{SCNb}$ ) samples 
showed very small roughness, below $30 \mathrm{~nm}$, after the BCP treatment. The very smooth surface together with less grain boundaries could potentially lead to a reduction of the residual resistance. Investigations of a $\mathrm{SCNb} 1.3$ $\mathrm{GHz}$ TESLA cavity fabricated at DESY are continuing at TJNAF to find minimum thickness of the total removed layer (minimum BCP) which provides high gradient and high quality factor. The preliminary, but very promising, result obtained at TJNAF is shown in Fig. 14. The single cell SCNb TESLA cavity was tested at $2 \mathrm{~K}$ after only a 80 $\mu \mathrm{m}$ layer was removed by the BCP treatment [24]. Both the gradient of $35.7 \mathrm{MV} / \mathrm{m}$ and $Q_{0}=10^{10}$ fulfill new spec of the ILC accelerator. This result compares favorably to the average removal of $280 \mu \mathrm{m}$ needed to reach such a gradient and $Q_{0}$ in the TESLA cavities with the standard EP treatment.

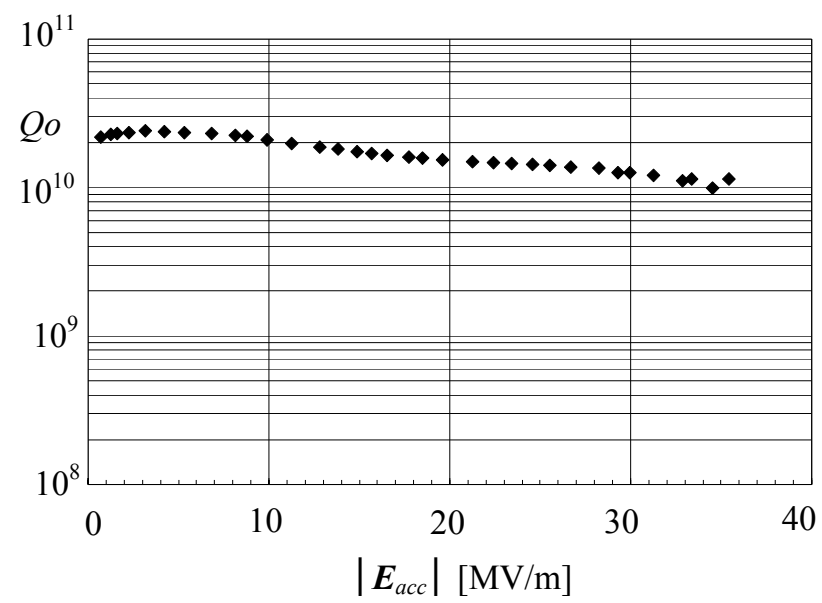

Figure 14: Test result for the single-cell BGNb TESLA cavity after the $80 \mu \mathrm{m}$ layer was removed by BCP. Courtesy P. Kneisel.

\section{FINAL REMARKS}

The presented material shows the complexity of the implementation of superconductivity in the accelerator technology required for future projects. The author does not aim to present all aspects of the field in depth; the intention was to give a flavor of the tremendous progress which has been achieved by the worldwide community over the past decades to enable new generations of unique facilities for experiments in many areas of science.

\section{ACKNOWLEDGMENT}

I want to express my gratitude to Dr. P. Kneisel and Dr. A. Burill for their valuable comments and corrections of the manuscript.

\section{REFERENCES}

[1] H. Schwettman et al., International Advances in Cryogenic Engineering, New York, Plenum Press, 1965 , p. 88.

[2] I. Weissman, J. Turneaure, Appl. Phys. Lett. 13, 390 (1968).

[3] Y. Kimura, "TRISTAN The Japanese ElectronProton Colliding Beam Project" Proceedings $11^{\text {th }}$ Int.
Conf. on High Energy Accelerators, CERN, 144 (1980).

[4] E. Picasso, "The LEP Project" - EPAC88, Proc. European Particle Accelerator Conference, 7-11 July 1988, Rome, Italy.

[5] C. W. Leemann, "The CEBAF Superconducting Accelerator: An Overview," CEBAF-PR-86-003, and Proceedings of the LINAC-86, June 2-6, 1986, SLAC, Stanford, CA.,

[6] HERA-Proposal, DESY-Report HERA-81-10, July 1981 Hamburg, FRG

[7] SNS CDR, http://www.ornl.gov/ nsns/, June 1997, ORNL, Oak Ridge, TN, USA.

[8] Editors R. Brinkmann et al., "TESLA TDR", TESLA-Report-2001-23, DESY, March 2001, Hamburg, FRG.

[9] ILC Baseline Conceptual Design, http://www.linearcollider.org/wiki/, 2005.

[10] Editors M. Altarelli et al., "TDR European XFEL", DESY Report 2006-97, July 2006, DESY Hamburg.

[11] S. L. Smith, "A review of ERL prototype experience and light source design challenges", Proceedings EPAC06, 26-30 June, 2006, Edinburgh UK,

[12]BESSY Soft X-FEL, Technical Design Report, March 2004, BESSY Berlin, FRG.

[13]D. Clarke et al., "4GLS A Fourth Generation Light Source that for the biomedical scientist is more than a laser and more than a storage ring", Proceedings of the Society of Photo-Optical Instrumentation Engineers; Vol. 4633; pp 233-242; 2002.

[14] E. Haebel, A. Mosnier, J. Sekutowicz, "Cavity Shape Optimization for a Superconducting Linear Collider", HEACC, Vol. 2, Hamburg, 1992.

[15] R. Collin, "Foundation for Microwave Engineering", McGraw-Hill, 2-nd edition, USA, 1992, ISBN: 0-07112569-8.

[16]P. Wilson, "Transient Beam Loading in ElectronPositron Storage Rings“, CERN-ISR-TH/78-23, 1978, CERN, Geneva, Switzerland.

[17]D. Nagel, E. Knapp, and B. Knapp, Rev. Scientific Instruments, 38, 1583 (1967).

[18] J. Sekutowicz, Beam Dynamics Newsletter, ICFA, No 39, April 2006.

[19] J. Sekutowicz et al., "Low Loss Cavity for the 12GeV CEBAF Upgrade“, JLAB, TN-02-023, June 2002, VA, USA.

[20] J. Sekutowicz et al., "Design of a Low Loss SRF Cavity for the ILC", APS, Proceedings PAC06, May 2005, Knoxville, TN, USA.

[21]R. Geng, "Review of New Shapes for Higher Gradients", Proceedings SRF2005 Workshop, July 10-15, 2005, Cornell University, Ithaca, NY, USA.

[22] V. Shemelin et al., "Optimal Cells for TESLA Accelerating Structure”, NIM A 496 (2003) 1-7.

[23] Y. Chen, J. Sekutowicz, Y. Wei, "A Different Tuning Method for Accelerating Cavities", Proc. $4^{\text {th }}$ Workshop on SRF'89, 849-858, August 1989, Tsukuba, Japan.

[24] P. Kneisel, private communication, 2006. 